\title{
O uso da técnica cognitiva substituição por imagem positiva no manejo do craving em dependentes de crack
}

The use of cognitive technique replacement by positive image in the management of craving for crack-cocaine-dependent patients

Mirian Pezzini dos Santos', Marcelo Rossoni da Rocha², Renata Brasil Araujo²

\section{RESUMO}

Objetivo: $O$ estudo visa avaliar a técnica cognitiva substituição por imagem positiva (SIP) no manejo do craving em dependentes de crack. Métodos: Ensaio clínico do tipo quase-experimental de análise quantitativa. Amostra por conveniência, composta por 34 homens dependentes de cocaína/crack. Tinham o crack como a droga de escolha, haviam utilizado essa substância pela última vez entre 10 e 20 dias antes do início do tratamento. Os instrumentos aplicados foram: Cocaine Craving Questionnaire-Brief (CCQB), Escala Analógica Visual (EAV) para avaliar o craving e o quanto a técnica ajudou e uma ficha com dados sociodemográficos (FSD). Foram realizadas intervenções individuais. Inicialmente, foram aplicados o CCQB, a EAV e a FSD. Depois, os pacientes foram expostos a objetos relacionados ao uso do crack e foram reaplicados os instrumentos CCQB e EAV para mensuração da fissura. A seguir, foi realizada a técnica cognitiva substituição por imagem positiva e foram aplicados, pela terceira vez, o CCQB e a EAV. Resultados: Os resultados desta pesquisa demonstraram uma redução dos escores do CCQB e da EAV pela técnica cognitiva SIP em uma amostra cujo

\section{Palavras-chave}

Cocaína crack, craving, fissura, terapia cognitiva. perfil corresponde ao padrão geral dos usuários de crack. Conclusão: Este estudo, apesar de algumas limitações metodológicas, sugere que a técnica cognitiva substituição por imagem positiva pode ser uma estratégia efetiva no manejo do craving em dependentes de crack.

\begin{abstract}
Objective: The objective of this study was to verify the effectiveness of cognitive technique replaced by positive image in the management of craving for crack-cocaine-dependent patients. Methods: This is a quasi-experimental clinical trial using a quantitative analysis. The convenience sample comprised 34 crack-cocaine-dependent males. These subjects had cocaine as their drug of choice, having used this substance between 10 and 20 days prior to the beginning of the treatment. The instruments used were: the Cocaine Craving QuestionnaireBrief (CCQB), the visual analogue scale (VAS) to assess craving and how the technique helps to decrease it, and a form containing sociodemographic (SDF). Individual interventions were conducted with the administration of the CCQB, the VAS, and the SDF. After that, patients
\end{abstract}

1 Escola de Saúde Pública - Hospital Psiquiátrico São Pedro, Porto Alegre, RS. 2 Hospital Psiquiátrico São Pedro, Porto Alegre, RS.

Endereço para correspondência: Mirian Pezzini dos Santos

Av. Sete de Setembro, 594, ap. 402 99010-121 - Passo Fundo, RS, Brasil

E-mail:mirianpezzini@yahoo.com.br 


\section{Keywords}

Crack-cocaine, craving,

cognitive therapy. were exposed to objects related to the use of crack to measure craving and the same instruments were administered again. Next, cognitive technique was performed by replacing positive image for 5 minutes and were applied for the third time the instruments CCQB and VAS.

Results: Our findings demonstrated a decrease in the scores on the CCQB and on the VAS after the cognitive technique for positive image replacement in the sample whose profile matches the general pattern of crack users. Conclusion: In spite of some methodological limitations, the present study suggests that the cognitive technique for positive image replacement is effective in the management of craving for crack-cocaine-dependent patients.

\section{INTRODUÇÃO}

No Brasil, dados epidemiológicos recentes mostram que o uso de cocaína/crack vem crescendo nos últimos anos entre os estudantes do ensino médio e fundamental, bem como entre os pacientes que procuram atendimento nas clínicas especializadas ${ }^{1,2}$. De acordo com II Levantamento Domiciliar sobre o uso de Drogas no Brasil, realizado pelo Centro Brasileiro de Informações sobre Drogas Psicotrópicas (Cebrid), constatou-se que a prevalência de uso na vida de cocaína nas 108 maiores cidades do país foi de 2,9\%, e, quanto ao crack, o uso na vida foi de 1,5\%3.

A cocaína é um alcaloide natural, extraído da planta Erythroxylon coca, estimulante do sistema nervoso central (SNC) e anestésico local. Estimula o SNC por meio do bloqueio da recaptação de dopamina, serotonina e noradrenalina nas sinapses ${ }^{4,5}$. O crack é um derivado da cocaína (do cloridrato de cocaína ou de sua pasta básica), a qual é misturada com outras substâncias, como o bicarbonato de sódio, transformando-se em "pedras", que são fumadas em cachimbos. Nessa forma de uso (via pulmonar), possui início de ação quase instantâneo (cerca de 10 segundos) e seu efeito estimulante tem duração de cerca de 10 minutos. Após esse período, o usuário passa por intensa depressão do SNC, que causa então os sintomas de abstinência e craving ${ }^{6,7}$.

O craving ou fissura, entendido como o desejo intenso por uma substância, é um dos pontos importantes no tratamento de dependentes químicos ${ }^{8}$. A sensação de cra- $^{-}$ ving possui componentes fisiológicos, cognitivos, afetivos e comportamentais, que, muitas vezes, acabam levando ao retorno do uso da substância em pacientes que se encontravam abstinentes 9 . Por causa da importância do craving no tratamento desses pacientes, muitas medicações vêm sendo testadas, porém ainda com resultados conflitantes e inconclusivos. Assim, o manejo do craving continua sendo essencialmente não farmacológico ${ }^{10,11}$.

Entre as psicoterapias para o tratamento de dependentes químicos, a que demonstra mais evidências de efetividade é a terapia cognitivo-comportamental (TCC), na qual são utilizadas técnicas para o manejo do craving ${ }^{9}$. Segundo o modelo cognitivo de Beck ${ }^{12}$ sobre o processo das recaídas, os estímulos de risco, que podem ser situações externas (como lugares ou objetos) e/ou internas (como depressão, irritabilidade ou sensação de bem-estar), ativarão crenças centrais dos pacientes e crenças a respeito do uso de drogas (crenças aditivas). Essas crenças, por sua vez, ativam pensamentos automáticos que desencadearão os sintomas de craving. Os sintomas fisiológicos de craving, geralmente vivenciados como forte ansiedade, "acionam" então crenças aditivas permissivas (como "usarei apenas um pouco") que podem levar ao uso da substância13. Juntamente com essas crenças aditivas, que estão associadas à busca do prazer (ou alívio do desprazer) e sensações de bem-estar, o paciente pode apresentar também crenças de controle (como: "terei prejuízos com o uso"), que podem, assim, diminuir a necessidade de consumir a substância psicoativa ${ }^{14}$.

Como o craving é considerado uma experiência individual, necessita, assim, de um planejamento personalizado quanto a seu manejo para cada paciente ${ }^{15}$. Muitas técnicas de manejo do craving são utilizadas. Na TCC, um dos pontos no tratamento desses pacientes é atenuar as crenças aditivas e reforçar suas crenças de controle ${ }^{16}$. Muitas técnicas cognitivas e comportamentais podem ser apreendidas pelos pacientes em busca do manejo do craving, as quais são conhecidas como habilidades de enfrentamento ${ }^{17,18}$. A maioria dessas técnicas é estudada e citada com sucesso em pacientes alcoolistas e dependentes de nicotina ${ }^{18,19}$, o que leva a serem utilizadas também no manejo do craving para outras substâncias ${ }^{20,21}$.

Pesquisas em pacientes alcoolistas têm demonstrado, como um ponto significativo na manutenção da abstinência, o componente cognitivo no enfrentamento ${ }^{15}$. Uma técnica imagística, amplamente utilizada e citada por vários autores cognitivo-comportamentais, entre eles Monti e Marlatt 15,19,20, é a chamada substituição por imagem positiva (SIP). Nessa técnica, o paciente faz uma visualização de si próprio adquirindo os benefícios que terá em decorrência do abandono da substância ou da manutenção da abstinência ${ }^{19,20}$. A visualização pode ser utilizada nas mais variadas ocasiões e patologias psiquiátricas; apresenta a característica de induzir a criação de imagens mentais que podem ser invocadas nas mais diversas situações e com vários objetivos ${ }^{22}$.

Na dependência química, a técnica SIP estimula o paciente a avaliar cognitivamente os benefícios da abstinência 
e, dessa forma, fortalece sua autoeficácia, ou seja, a percepção de sua habilidade de desempenhar de forma eficaz o manejo do craving e, assim, não utilizar a substância ${ }^{15}$.

O não manejo do craving é um dos principais fatores que levam ao retorno do uso da substância, dado esse de significativa importância no tratamento da dependência química?

Atualmente, dispomos de poucas pesquisas sobre esse tema em dependentes de cocaína/crack, com um número ainda menor quando consideramos a avaliação de tratamento não farmacológico. Assim, o presente estudo apresenta relevância em virtude da escassez de estudos nessa área e visa, de alguma forma, incentivar outros estudos que avaliem o manejo do craving em dependentes químicos de cocaína/ crack em suas variadas possibilidades. Ele tem como objetivo avaliar o uso da técnica cognitiva SIP no manejo do craving nesses pacientes.

\section{MÉTODOS}

\section{Delineamento}

Este estudo caracteriza-se como um ensaio clínico do tipo quase-experimental de análise quantitativa. Os dados foram coletados no período de abril do ano 2011 a outubro do mesmo ano, em uma unidade de desintoxicação masculina, por meio de entrevista clínica individual, com duração de cerca de 40 minutos cada.

\section{Participantes}

A amostra foi coletada "por conveniência"; foram avaliados 34 pacientes do sexo masculino, entre 18 e 65 anos de idade. Os critérios de inclusão para o estudo foram: ter diagnóstico de dependência de cocaína/crack, segundo os critérios da quarta edição do Manual de Diagnóstico e Estatística dos Transtornos Mentais (DSM-IV, American Psychiatric Association, 2002); estar em abstinência por um período de, no mínimo, 10 dias e, no máximo, 20 dias e aceitar participar da pesquisa. Os critérios de exclusão foram: ter sintomas psicóticos, ter qualquer evidência clínica de retardo mental ou doença neurológica que fosse incapacitante cognitivamente para uma adequada aplicação da intervenção e preenchimento dos questionários.

\section{Instrumentos}

Os instrumentos utilizados foram: (1) Cocaine Craving Questionnaire-Brief (CCQB) validada ${ }^{23}$ e adaptada para o português ${ }^{24}$ para avaliar e quantificar a presença de craving por cocaína/crack. Essa escala é composta por 10 questões e pode ser avaliada pelo seu total de pontos ou por seus dois fatores: o fator 1 refere-se ao craving propriamente dito e o fator 2 , à percepção que o indivíduo tem quanto ao descontrole do uso do crack. Pode ser graduada em grau mínimo, leve, moderado e grave. Os pontos de corte da escala são: grau mínimo (CCQB total: 0 a 11; fator 1: 0 a 7 e fator 2: 0 a 2 pontos); grau leve (CCQB total: 12 a 16; fator $1: 8$ a 9 e fator 2: 3 a 4 pontos); grau moderado (CCQB total: 17 a 22; fator 1 : 10 a 11 e fator 2: 5 a 6 pontos); grau grave (CCQB total: 23 ou mais pontos; fator 1: 12 ou mais pontos e fator 2: 7 ou mais pontos); (2) Escalas Analógicas Visuais (EAV) de 0 a 10 para quantificar a fissura (sendo 0 igual a nenhuma fissura e 10, muita fissura) e para avaliar o quanto a técnica SIP ajudava no manejo do craving (0, não ajuda e 10, ajuda muito); (3) ficha de dados demográficos e referentes ao uso da substância (FSD) para identificar características gerais da amostra e padrão do uso da substância; (4) parafernália do crack (cachimbos, pedras simuladas, cinza de cigarro e isqueiro) como estímulo para indução do craving.

\section{Procedimentos}

\section{Aspectos éticos}

O estudo foi realizado após aprovação do Comitê de Ética e Pesquisa do Hospital Psiquiátrico São Pedro (HPSP). Os participantes leram e assinaram um Termo de Consentimento Livre e Esclarecido, por meio do qual foram explicitadas as implicações referentes à pesquisa.

\section{Coleta de dados}

Os participantes foram avaliados pelo terapeuta individualmente, em uma sala adequada, com pouca interferência externa. Foram orientados, inicialmente, sobre o objetivo da pesquisa, sobre o craving e a SIP como um instrumento de enfrentamento dele.

No primeiro momento, foram aplicados os seguintes instrumentos: CCQB, EAV para avaliação do craving e ficha de dados demográficos (FSD). Após a aplicação dos instrumentos, os participantes foram expostos à parafernália relacionada ao crack por um tempo de 3 minutos. Após a exposição e o manuseio do material pelos pacientes, que tinham como objetivo a indução de craving, foram reaplicados os instrumentos CCQB e EAV com a intenção de avaliar a presença e a intensidade do craving. Na sequência, os participantes foram orientados a exercitar a técnica cognitiva SIP por meio da seguinte frase: "pense em todas as coisas boas que aconteceriam se você se mantivesse sem usar o crack nessa situação". Durante a aplicação da técnica, pelo tempo de 5 minutos, os pacientes eram estimulados a fechar os olhos e imaginar-se na situação positiva citada, sendo estimulados a descrever e a refletir melhor sobre os sentimentos positivos que surgiam ${ }^{14}$. Após a aplicação da técnica, o paciente respondia os instrumentos pela terceira vez (CCQB e EAV) para, assim, avaliar e quantificar novamente o craving. Por último, os entrevistados responderam a outro instrumento, uma EAV, sobre, segundo a impressão do paciente, o quanto a técnica estudada e treinada ajudou no manejo do craving. 


\section{Análise de dados}

Os dados foram analisados por meio do programa Statistical Package for the Social Sciences (SPSS) versão 17.0 e tratados estatisticamente, sendo feita uma análise descritiva e de frequência dos dados sociodemográficos e referentes ao padrão de consumo de substâncias psicoativas. Foi utilizado o teste $t$ de Student para amostras pareadas para o estudo inferencial. O nível de significância foi de 5\%.

\section{RESULTADOS}

Foram avaliados 34 homens, com idade média de 26,9 anos $(\mathrm{DP}=7,19 ; 18-43)$, a maioria deles $(70,6 \%)$ com história psiquiátrica familiar positiva para doenças psiquiátricas e com média de 8,44 anos de estudo ( $D P=2,12 ; 5-13)$. Em relação ao estado civil, $91 \%$ eram solteiros, $6 \%$, casados e $3 \%$, separados. Todos tinham como droga de escolha o crack, assim 100\% dos entrevistados eram dependentes de crack, 79\%, dependentes de nicotina, 17\%, dependentes de maconha e $6 \%$, dependentes de álcool. A tabela 1 demonstra outros dados sociodemográficos e perfil de uso de substâncias psicoativas.

Tabela 1. Perfil de uso de substâncias psicoativas $(n=34)$

\begin{tabular}{lcccc}
\hline \multicolumn{1}{c}{ Variáveis } & Média & Desvio-padrão & Mín & Máx \\
\hline Tempo de hospitalização (dias) & 13,3 & 3,11 & 10 & 20 \\
Último uso de crack (dias) & 15,5 & 3,86 & 10 & 21 \\
Idade de início do uso do crack (anos) & 20,6 & 6,56 & 12 & 39 \\
Idade de início do uso da cocaína (anos) & 16,6 & 4,69 & 0 & 27 \\
Idade de início do uso do álcool (anos) & 13,6 & 2,48 & 7 & 18 \\
Idade de início do uso da maconha (anos) & 15,1 & 3,59 & 10 & 30 \\
Idade de início do uso da nicotina (anos) & 15,3 & 5,42 & 7 & 39 \\
Consumo semanal de crack (gramas) & 16,4 & 16,7 & 2 & 67 \\
Consumo semanal de álcool (unidades & 13,8 & 34,2 & 0 & 180 \\
internacionais de álcool*) & & & & \\
Consumo semanal de maconha (baseados) & 10,8 & 42,9 & 0 & 250 \\
\hline
\end{tabular}

* 1 unidade internacional equivale a 10 gramas de álcool.

Quando questionados quanto ao perfil de violência, 9\% dos entrevistados diziam-se pessoas violentas antes do início do uso do crack; após início do uso da substância, 44\% dos pacientes afirmaram ter apresentado episódios de violência, com agressões físicas a terceiros. Dos pacientes avaliados, 56\% relataram problemas com a justiça, entre eles: 58\% por motivo de furto/roubo, $16 \%$ por envolvimento com tráfico de drogas, $11 \%$ devido à Lei Maria da Penha, $5 \%$ por tentativa de homicídio e lesão corporal, 5\% por desordem e 5\% por problemas de posse de drogas.

A tabela 2 demonstra as comparações, segundo o teste $t$ de Student para amostras pareadas, nas médias do craving antes (tempo A) e depois da exposição à parafernália do crack (tempo B), e nas médias do craving antes (Tempo B) e depois da aplicação (tempo C) da técnica cognitiva estudada (SIP). Também foram analisadas as médias das EAV para avaliar o craving antes e depois da exposição para o crack, e após aplicação da SIP.

Tabela 2. Comparação das médias e desvios-padrão de craving antes e depois da exposição para o crack e do craving antes e depois da aplicação da técnica cognitiva SIP $(n=34)$

\begin{tabular}{lcccc}
\hline Variáveis & Média & Desvio-padrão & $\mathbf{T}$ & $\mathbf{p}$ \\
\hline CCQB fator 1 - tempo A & 10,5 & 3,93 & $-8,00$ & $<0,001$ \\
CCQB fator 1 - tempo B & 26.6 & 12,9 & & \\
CCQB fator 2 - tempo A & 2,97 & 1,96 & $-11,9$ & $<0,001$ \\
CCQB fator 2 - tempo B & 10,0 & 3,05 & & \\
CCQB total - tempo A & 13,5 & 5,37 & $-10,1$ & $<0,001$ \\
CCQB total - tempo B & 36,7 & 14,8 & & \\
CCQB fator 1 - tempo B & 26,6 & 12,9 & 22,0 & $<0,001$ \\
CCQB fator 1 - tempo C & 8,94 & 1,55 & & \\
CCQB fator 2 - tempo B & 10,0 & 3,05 & 8,68 & $<0,001$ \\
CCQB fator 2 - tempo C & 2,32 & 0,53 & & \\
CCQB total - tempo B & 36,7 & 14,8 & 10,5 & $<0,001$ \\
CCQB total - tempo C & 11,3 & 1,76 & & \\
EAV - tempo A & 0,65 & 1,27 & $-10,1$ & $<0,001$ \\
EAV - tempo B & 5,6 & 3,02 & & \\
EAV - tempo B & 5,6 & 3,02 & 10,2 & $<0,001$ \\
EAV - tempo C & 0,53 & 0,82 & & \\
\hline
\end{tabular}

CCQB: Cocaine Craving Questionnaire Brief; EAV: Escala Analógica Visual; SIP: técnica cognitiva substituição por imagem positiva; A: antes da exposição/indução de craving; B: após indução de craving; C: após aplicação da SIP.

Em relação à EAV aplicada após a aplicação da SIP, em que foi mensurado, pela opinião dos pacientes, o quanto a técnica estudada auxiliou no manejo do craving, a média referida para a SIP foi de 9,29 (DP = 1,03), com avaliação de no mínimo nota 6 e no máximo nota 10.

\section{DISCUSSÃO}

A amostra deste estudo demonstra a predominância do consumo de crack por adultos jovens, com baixa escolaridade, solteiros, com início do uso de álcool e de nicotina durante a adolescência. Eram dependentes de outras substâncias psicoativas associados ao crack e tinham história familiar psiquiátrica positiva para doenças psiquiátricas, fatores esses comuns em dependentes de crack e também observados em outros estudos, 2 .

Pode-se perceber um aumento significativo de episódios de violência após início do uso da substância, subindo de 9\% para $44 \%$, fato esse observado em outros estudos, o que demonstra que usuários de crack estão mais expostos a situações de violência e envolvimento com o tráfico de drogas e roubos ${ }^{25,26}$. A maioria dos entrevistados afirmava ter dificuldades no manejo da fissura pelo crack, o que demonstra não 
somente o grau de dependência que essa droga provoca, mas também a importância de uma orientação para o manejo adequado do craving para esses pacientes.

$\mathrm{Na}$ intervenção realizada, houve significativa indução do craving por meio dos escores do CCQB e da EAV com a exposição a materiais relacionados ao crack (parafernália do (rack). Alguns participantes apresentaram menor indução do craving após a exposição, fato que pode ter ocorrido tanto pela dificuldade de reconhecer em si o craving como pelo receio de ter sua alta adiada, já que se encontravam em período de internação. Alguns pacientes relataram que sentiram o craving de maneira atenuada, pois se encontravam em ambiente protegido, continente e afastado do acesso. Em outros pacientes, pelo contrário, pode-se identificar intensa fissura, percebidos também por sintomas físicos como tremores, sudorese e inquietação. Esses fatos também foram observados em estudo realizado com dependentes de crack, no qual, de forma similar, foram avaliados indução, mensuração e manejo do craving ${ }^{7}$.

Após a aplicação da técnica cognitiva SIP, percebeu-se significativa redução do craving, mensurada por meio das escalas CCQB e EAV. Durante a intervenção, também se percebeu facilidade de aplicação da SIP nos pacientes, bem como resposta significativa deles na avaliação da técnica, considerada de grande auxílio para diminuição do craving, medida essa realizada por meio de EAV. Em estudo realizado com alcoolistas, entre outras técnicas cognitivas e comportamentais avaliadas, a SIP foi citada como uma técnica de auxílio para redução do craving $^{19}$. Em recente estudo de caso que avaliou o tratamento de exposição a estímulos com paciente dependente de crack, a SIP foi observada como uma estratégia de enfrentamento efetiva e uma das técnicas de escolha pelo paciente no manejo do craving ${ }^{27}$.

No presente estudo, durante a aplicação da SIP, quando estimulados a refletirem sobre os benefícios de manterem-se abstinentes, a maioria dos pacientes relatou como principais benefícios o retorno de um bom relacionamento familiar, adequadas realizações de atividades laborais e um aumento de poder aquisitivo e a maneira de como seriam vistos na sociedade sem a droga (com respeito e confiança). A técnica estudada fortalece para o paciente as vantagens de manterem-se abstinentes e a possibilidade de realizações concretas caso se mantenham abstinentes.

A SIP é um instrumento terapêutico fácil de ser entendido e aplicado nos pacientes, podendo ser usada pelos diversos profissionais da área da saúde que atendem pacientes dependentes de cocaína/crack.

Neste estudo, foram observadas algumas limitações, entre elas não terem sido avaliados os diagnósticos comórbidos, o fato de a amostra ser pequena e "por conveniência", a ausência de pacientes controles e possíveis comparações com outras técnicas cognitivo-comportamentais, bem como a não individualização do material utilizado para a exposição, o que talvez induzisse um craving mais intenso ${ }^{18}$. O fato de ser o terapeuta quem aplica a SIP e também as escalas para quantificar tanto a fissura quanto a ajuda provida pela SIP pode ter apresentado alguma influência nos resultados, pois o paciente pode tender para o agrado ao seu terapeuta.

\section{CONCLUSÃO}

A intervenção realizada neste estudo proporcionou a avaliação da técnica cognitiva SIP como uma estratégia de manejo do craving, técnica fácil de ser aplicada e treinada com os pacientes, o que pode ser muito útil no contexto clínico e psicoterápico de dependentes de crack.

A técnica estudada, se realizada adequadamente, em momentos de risco de craving ou do craving instalado, pode contribuir com o aumento do senso de autoeficácia desses pacientes, podendo, assim, melhorar a sua capacidade de lidar com essa emoção de forma a prevenir recaídas.

Acreditamos, assim, que a técnica SIP usada na TCC 13,15,16,19,22,27 representa um importante instrumento terapêutico para o manejo do craving pelo crack, uma droga que apresenta elevados índices de dependência e de recaídas.

\section{CONTRIBUIÇÕES INDIVIDUAIS}

Mirian Pezzini dos Santos - Contribuiu na autoria, elaboração do artigo, revisão da literatura, coleta de dados e análise estatística.

Renata Brasil Araújo - Contribuiu na orientação, elaboração e auxílio estatístico.

Marcelo Rossoni da Rocha - Contribuiu na coleta de dados e revisão da literatura.

Todos os autores contribuíram diretamente na elaboração do artigo.

\section{REFERÊNCIAS}

1. Laranjeira R, Silveira DX, Formigoni ML, Ferri CP, Dunn J. Crack cocaine: an increase in use among patients attending clinics in São Paulo: 1990-1993. Subst Use Misuse. 1996;31:519-27.

2. Oliveira LG, Nappo AS. Caracterização da cultura de crack na cidade de São Paulo: padrão de uso controlado. Rev Saude Publica 2008;42(4):664-71.

3. Carlini EA. II Levantamento domiciliar sobre 0 uso de drogas psicotrópicas no Brasil. São Paulo: Centro Brasileiro de Informações sobre Drogas Psicotrópicas (Cebrid), Departamento de Psicobiologia, Escola Paulista de Medicina, Universidade Federal Paulista; 2005.

4. World Health Organization. Neuroscience of psychoactive substance use and dependence. Geneva: WHO; 2004.

5. Romano M, Ribeiro M, Marques ACPR. Abuso e dependência da cocaína. Associação Brasileira de Psiquiatria. Projeto Diretrizes - Associação Médica Brasileira e Conselho Federal de Medicina; 2002

6. Seibel SD, Toscano Jr. A. Dependência de drogas. São Paulo: Atheneu; 2001. 
7. Zeni TC, Araújo RB. 0 relaxamento respiratório no manejo do craving e dos sintomas de ansiedade em dependentes de crack. Rev Psiquiatr Rio Gd Sul. 2009;31(2):116-9.

8. Araújo RB, Oliveira MS, Moraes JF, Pedroso RS, Port F, de Castro MG. Validation of the Brazilian version of Questionnaire of Smoking Urges-Brief. Rev Psiq Clín. 2007;34(4):166-75.

9. Araújo RB, Oliveira MS, Pedroso RS, Miguel AC, Castro MG. Craving e dependência química: conceito, avaliação e tratamento. J Bras Psiquiatr. 2008;57(1):57-6.

10. Witters NW, Pulvirenti L, Koob GF, Gillin JC. Cocaine abuse and dependence. J Clin Psychopharmacol. 1995;15(1):63-78.

11. Focchi GRA, Leite MC, Scivoletto S. Utilização do agonista dopaminérgico pergolida no tratamento da "fissura" por cocaína. Rev Bras Psiquiatr. 2001;23(4):188-94.

12. Knapp P, Beck AT. Fundamentos, modelos conceituais, aplicações e pesquisa da terapia cognitiva. Rev Bras Psiquiatr. 2008;30(Supl II):S54-64.

13. Beck A, Newman C, Wright F. Terapia cognitiva de las drogodependencias. Barcelona: Ediciones Paidos Iberica; 2000.

14. Ramos SP, Bertolote JM, organizadores. Alcoolismo hoje. Porto Alegre: Artes Médicas; 1997.

15. Marlatt AG, Donavan DM. Prevenção de recaída: estratégias de manutenção no tratamento de comportamentos adictivos. Porto Alegre: Artmed; 2009.

16. Knapp P, et al. Terapia cognitivo-comportamental na prática psiquiátrica. Porto Alegre: Artmed; 2004.

17. Knapp P, Beck AT. Fundamentos, modelos conceituais, aplicações e pesquisa da terapia cognitiva. Rev Bras Psiquiatr. 2008;30(Supl II):S54-64.
18. Rohsenow DJ, Monti PM, Rubonis AV, Gulliver SB, Colby SM, Binkoff JA, et al. Cue exposure with coping skills training and communication skills training for alcohol dependence: 6and 12-month outcomes [research report]. Addiction. 2001;96:1161-74.

19. Monti PM, Rohsenow JD. Coping-skills training and cue-exposure therapy in the treatment of alcoholism. Alcohol Res Health. 1999;23(2):107-15.

20. Monti PM, Kadden RM, Rohsenoww DJ, Cooney NL, Abrams DB. Tratando a dependência do álcool: um guia de treinamento das habilidades de enfrentamento. $2^{a}$ ed. São Paulo: Roca; 2005.

21. Monti PM, Rohsenow DJ. Coping-Skills training and cue-exposure therapy in the treatment of alcoholism. Alcohol Res Health. 1999;23(2):107-15.

22. Rangé $B$ (org.). Psicoterapias cognitivo-comportamentais: um diálogo com a psiquiatria. Porto Alegre: Artmed; 2001.

23. Sussner BD, Smelson DA, Rodrigues S, Kline A, Losonczy M, Ziedonis D. The validity and reliability of a brief measure of cocaine craving. Drug Alcohol Depend. 1999;83:233-7.

24. Araújo RB, Pedroso RS, Graça MGT. Adaptação transcultural para 0 idioma português do Cocaine Craving Questionnaire - Brief. Rev Psiq Clín. 2010;37(5):195-8.

25. Ferreira Filho OF, Turchi MD, Laranjeira R, Castelo A. Perfil sociodemográfico e de padrões de uso entre dependentes de cocaína hospitalizados. Rev Saude Publica. 2003;37(6):751-9.

26. Chalub M, Telles LEB. Alcohol, drugs and crime. Rev Bras Psiquiatr. 2006;28(Supl II):S69-73.

27. Araújo RB, Balbinot AD, Castro MGT, Rocha MR, Miguel SRPS, Cohen M. Tratamento de exposição a estímulos e treinamento de habilidades como coadjuvantes no manejo do craving em um dependente de crack. Trends Psychiatry Psychother. 2011;33(3):181-8. 